\title{
Global Fixed-Time Stabilization for Chained Nonholonomic Systems via Output Feedback Control
}

\section{Xiandong Chen}

Shandong University

Xianfu Zhang ( $\sim$ zhangxianfu@sdu.edu.cn)

Shandong University https://orcid.org/0000-0001-9232-6099

\section{Research Article}

Keywords: Nonholonomic systems , Fixed-time stabilization , Output-feedback control , bi-limit homogeneous technique, Switching control

Posted Date: May 24th, 2021

DOl: https://doi.org/10.21203/rs.3.rs-364743/v1

License: (c) (1) This work is licensed under a Creative Commons Attribution 4.0 International License.

Read Full License 


\title{
Global Fixed-Time Stabilization for Chained Nonholonomic Systems via Output Feedback Control
}

\author{
Xiandong Chen · Xianfu Zhang
}

the date of receipt and acceptance should be inserted later

\begin{abstract}
This paper studies the fixed-time output feedback stabilization control problem for chained nonholonomic systems. By means of switching control and $b i$ limit homogeneous techniques, it is firstly constructed two fixed-time state feedback stabilizing controllers for the considered systems. Then, a new state observer with a formalized switching law is proposed to fixed-time estimate system states, where high-order terms are applied to get uniform convergence regardless of initial conditions and low-order terms are aimed to the exact convergence in finite time. Finally, based on bi-limit homogeneous technique and Lyapunov stability theorem, fixed-time output feedback stabilizing controllers, one of which is discontinuous with a specific switching control law and the other is continuous, are constructed and the fixed-time output feedback stabilization of the considered systems is thus guaranteed. An example is presented to show the feasibility of the proposed fixedtime output feedback stabilization control strategy.
\end{abstract}

Keywords Nonholonomic systems · Fixed-time stabilization · Output-feedback control · bi-limit homogeneous technique $\cdot$ Switching control

\section{Introduction}

Fixed-time control, whose stability guaranteed the settling time irrelevant to initial conditions firstly introduced in 24], has drawn an increasing attention in recent years [18,34. Fixed-time stabilization problems have been studied in many control systems, see 11 ,

X. Chen $\cdot$ X. Zhang $(\varangle)$

Control Science and Engineering, Shandong University, Ji'nan 250061, P.R. China

E-mail: zhangxianfu@sdu.edu.cn
13, 26, 31, 33 and the references therein. For example, through introducing implicit Lyapunov theorems for fixed-time stability analysis of nonlinear systems, fixedtime state feedback stabilizing controllers were designed in 26 for a chain of integrators. By backstepping method, 13. constructed decentralized fixed-time state feedback controllers to stabilize nonlinear interconnected systems. By means of recursive approach, a state feedback controller was proposed in 33 to study the fixed-time almost disturbance decoupling problem for a class of nonlinear systems. 11] investigated the global fixed-time state feedback stabilization of switched nonlinear systems with general powers. However, all the works mentioned above are based on designing state feedback controllers to achieve the fixed-time stabilization control.

Observer design, a classical control problem of control systems when part of states are available, has gotten a great variety of solutions $1,8,2128$. As a straightforward idea, how to design fixed-time observers for control systems has also attracted extensive attention $20,22,25,27$. Recently, a hybrid observer was proposed in 27 to fixed-time state estimation for a kind of linear systems. The $b i$-limit homogeneous technique introduced in [2] has shown that a fixed-time stable system is asymptotically stable firstly, and is also homogeneous with negative degree in 0-limit and homogeneous with positive degree in $\infty$-limit. By means of this technique, 22 studied fixed-time observer design problems for many kinds of nonlinear systems including linearizable systems up to input-output injection and uniformly observable systems. Moreover, through introducing implicit Lyapunov function approach, nonlinear dynamic observers were proposed in 20 to deal with fixed-time observation of a class of linear systems. Through proposing distributed observers, 77 investigated the distributed fixed-time consensus problem for 
heterogeneous multi-agent systems. However, the aforementioned results on fixed-time observer design have great challenges in being applied to construct fixed-time output feedback stabilizing controllers of nonlinear systems. To our knowledge, few works have achieved the fixed-time output feedback stabilization of control systems based on designing fixed-time observer. By means of $b i$-limit homogeneous technique, 29] proposed a fixedtime observer to achieve the fixed-time output feedback stabilization of double integrator systems. [19] studied the global fixed-time output feedback stabilization and estimation of a chain of integrators.

On the other hand, chained nonholonomic systems are one of the most fundamental systems in control theory and have many applications in practice, such as wheeled mobile robots and the knife edge [17,23. And for fixed-time control design, by means of switching control technique, [30] studied the fixed-time state feedback stabilization problem for chained nonholonomic systems. 5] dealt with the fixed-time state feedback stabilization problem through sliding mode control for chained nonholonomic systems. Through proposing distributed observers, the consensus tracking problem for multi-agent systems with chained nonholonomic dynamics was studied in 23. However, no work has been done for the fixed-time output feedback stabilization control of chained nonholonomic systems, and partly inspired by $10,23,29$, this paper focuses on this problem by means of switching control and bi-limit homogeneous technique.

The main contributions of this paper are as follows. Firstly, by synthesizing switching control and $b i$-limit homogeneous techniques, the fixed-time output feedback stabilization problem of chained nonholonomic systems is studied for the first time. Secondly, a unified framework for constructing two coupled fixed-time output feedback stabilizing controllers is proposed. In detail, apparently different from 29 only designing one controller, two coupled fixed-time output feedback stabilizing controllers, one of which is discontinuous combining with a switching law, and the other is continuous, are constructed simultaneously, which is more challenging. Moreover, the switching control law has a more complex structure and requires more sophisticated design skills than that of in [10]. Thirdly, by the aid of the discontinuous controller and the designed switching control law, a fixed-time observer is proposed to estimate system states in a fixed time, and then based on this observer, the control objective is thus achievement.

The rest of this paper is organized as follows. Problem preliminaries are given in Section 2. Main theoretical results on constructing a fixed-time observer and fixed-time output feedback stabilizing controllers are stated in Section 3. Convincing simulation is shown in Section 4. Finally, Section 5 concludes this paper.

\section{Preliminaries}

\subsection{Notations}

Throughout the paper, the argument of the functions will be omitted or simplified whenever no confusion arises from the context. For example, we denote $x(t)$, $\phi(x): \mathbb{R}^{n} \rightarrow \mathbb{R}$ and $\Phi(x): \mathbb{R}^{n} \rightarrow \mathbb{R}^{n}$ by $x, \phi(x)$ and $\Phi(x)$, respectively. $\mathbb{R}$ is the set of real numbers. For any given non-negative real number $\varrho, s \mapsto\lceil s\rfloor \varrho$ is a function defined as $\lceil s\rfloor^{\varrho}=|s|^{\varrho} \operatorname{sign}(s), \forall s \in \mathbb{R}$, where $\operatorname{sign}(s)$ denotes the standard sign function $([4,6])$. From the definition, $\frac{d\lceil s\rfloor^{\varrho}}{d s}=\varrho|s|^{\varrho-1},\lceil s\rfloor^{0}=\operatorname{sign}(s),\lceil s\rfloor=s$ and $\lceil s\rfloor^{2}=s|s|$ hold.

\subsection{Definitions and lemmas}

Consider the nonlinear system

$\dot{x}(t)=\Phi(x(t)), \quad t \geq t_{0}, \quad x\left(t_{0}\right)=x_{0}$,

with $x=\left(x_{1}, \ldots, x_{n}\right)^{T} \in \mathbb{R}^{n}$ being system states and $\Phi(x)$ denoting a possibly discontinuous vector field. If $\Phi(x)$ is discontinuous, the solution of (1) is understood in the sense of Filippov ( [9] $)$, and in this paper, the origin is assumed as an equilibrium point of system (1).

Let $r=\left(r_{1}, \ldots, r_{n}\right)^{T} \in \mathbb{R}^{n}$ be a weight vector with $r_{i}>0, i=1, \ldots, n$ and $\Lambda_{\lambda}^{r}(x)=\left(\lambda^{r_{1}} x_{1}, \ldots, \lambda^{r_{n}} x_{n}\right)$ be a dilation mapping with $\lambda>0$.

Definition 1 ( [3]) A function $\phi(x)$ is $r$-homogeneous with degree $k \in \mathbb{R}$ if $\phi\left(\Lambda_{\lambda}^{r}(x)\right)=\lambda^{k} \phi(x), \forall x \in \mathbb{R}^{n}, \forall \lambda>$ 0 . Similarly, a vector field $\Phi(x)$ is $r$-homogeneous with degree $k$ if its component $\Phi_{i}(x)$ is $r$-homogeneous of degree $k+r_{i}, i=1, \ldots, n$.

Definition 2 ( 2]) A function $\phi(x)$ is homogeneous in the $p$-limit $(p=0$ or $\infty)$ with $\left(r_{p}, k_{p}, \phi_{p}\right)$, in which $r_{p}=\left(r_{p, 1}, \ldots, r_{p, n}\right)^{T} \in \mathbb{R}^{n}$ is the weight vector, $k_{p}$ is the degree, and $\phi_{p}$ is the approximating function. If $\phi(x)$ is continuous, $\phi_{p}$ is continuous and not identically zero, and hence, for each compact set $C \in \mathbb{R}^{n} \backslash\{0\}$, $\lim _{\lambda \rightarrow p} \max _{x \in C}\left|\lambda^{-k_{p}} \phi\left(\Lambda_{\lambda}^{r_{p}}(x)\right)-\phi_{p}(x)\right|=0$ holds.

Definition 3 ( 2]) A vector field $\Phi(x)$ is homogeneous in the $p$-limit $(p=0$ or $\infty)$ with $\left(r_{p}, k_{p}, \Phi_{p}\right)$, in which $r_{p}=\left(r_{p, 1}, \ldots, r_{p, n}\right)^{T} \in \mathbb{R}^{n}$ is the weight vector, $k_{p}$ is the degree and $\Phi_{p}$ is the approximating vector field, if, for each $i, k_{p}+r_{p, i}>0$ and $\Phi_{i}(x)$ is homogeneous in the $p$-limit with $\left(r_{p}, k_{p}+r_{p, i}, \Phi_{p, i}\right)$. 
Definition 4 ( 2]) A function (or vector field) is homogeneous in the $b i$-limit if it is homogeneous in the 0 limit and homogeneous in the $\infty$-limit simultaneously.

Definition 5 ( [16]) The origin of system (1) is globally finite-time stable if it is globally asymptotically stable and there exists $T\left(x_{0}\right)>0, \forall x_{0} \in \mathbb{R}^{n}$, such that the trajectory stabilizes at the origin in $T\left(x_{0}\right)$.

Definition 6 ( 24] ) The origin of system (1]) is fixedtime stable if it is globally finite-time stable and there is a constant $T_{\max }>0$, such that $T\left(x_{0}\right) \leq T_{\max }, \forall x_{0} \in$ $\mathbb{R}^{n}$.

Lemma 1 ( [2]) For system (1), suppose that $\Phi(x)$ is a homogeneous vector field in the bi-limit with $\left(r_{0}, k_{0}, \Phi_{0}\right)$ and $\left(r_{\infty}, k_{\infty}, \Phi_{\infty}\right)$. If the origins of systems $\dot{x}=\Phi(x)$, $\dot{x}_{0}=\Phi_{0}(x), \dot{x}_{\infty}=\Phi_{\infty}(x)$ are globally asymptotically stable, then we have

1) The origin of system (1) is fixed-time stable as long as $k_{\infty}>0>k_{0}$ holds;

2) Let $d_{W_{0}}$ and $d_{W_{\infty}}$ be real numbers satisfying $d_{W_{0}}>$ $\max _{1 \leq i \leq n} r_{0, i}$ and $d_{W_{\infty}}>\max _{1 \leq i \leq n} r_{\infty, i}$. There exists a continuous, positive definite and proper function $W(x)$ such that $\frac{\partial W}{\partial x_{i}}$ is homogeneous in the bilimit with $\left(r_{0}, d_{W_{0}}-r_{0, i}, \frac{\partial W_{0}}{\partial x_{i}}\right)$ and $\left(r_{\infty}, d_{W_{\infty}}-\right.$ $\left.r_{\infty, i}, \frac{\partial W_{\infty}}{\partial x_{i}}\right)$ and $\frac{\partial W}{\partial x} \Phi(x)$ is negative definite.

\section{Main Results}

In this section, we first explicitly construct fixed-time state feedback stabilizing controllers by $b i$-limit homogeneous technique. Then, we propose an fixed-time state observer. Finally, the unmeasurable states in state feedback controllers is replaced with the estimate recovered from the observer to fixed-time stabilize the considered systems.

\subsection{Fixed-time state feedback control}

Throughout this paper, we consider a unicycle-type mobile robot given in Fig 1 w whose dynamic equations is

$\dot{x}_{l}=v \cos \theta, \quad \dot{y}_{l}=v \sin \theta, \quad \dot{\theta}=w$,

with $\left(x_{l}, y_{l}\right)$ denoting the position of the center of mass, $\theta$ being the heading angle, and $v$ and $w$ standing for the forward velocity and the angular velocity of the robot, respectively. Similar to [10, introducing the state transformation

$x_{0}=x_{l}, \quad x_{1}=y_{l}, \quad x_{2}=\tan \theta$,

$u_{0}=v \cos \theta, \quad u_{1}=w \sec ^{2} \theta$,

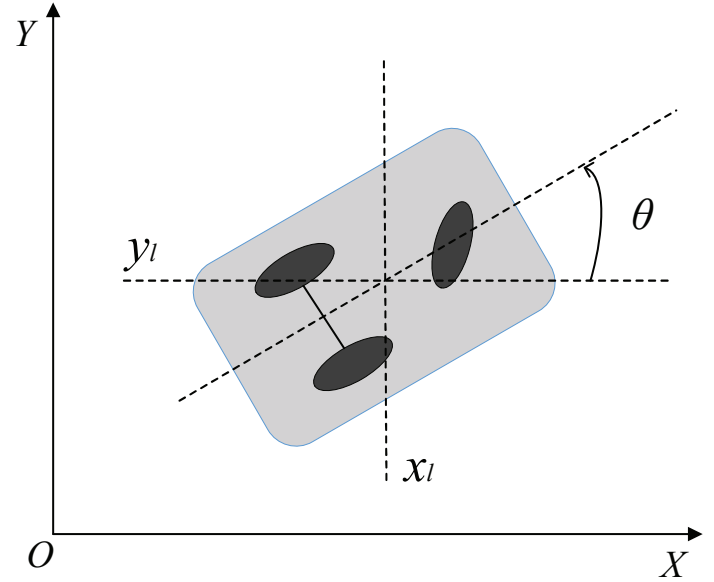

Fig. 1 The planar graph of a unicycle-type mobile robot.

system (2) is converted into the chained form

$\dot{x}_{0}=u_{0}, \quad \dot{x}_{1}=u_{0} x_{2}, \quad \dot{x}_{2}=u_{1}$,

with $\left(x_{0}, x^{T}\right)^{T}=\left(x_{0}, x_{1}, x_{2}\right)^{T} \in \mathbb{R}^{3}$ being system states, and $u_{0}, u_{1} \in \mathbb{R}, y=\left(x_{0}, x_{1}\right) \in \mathbb{R}^{2}$ being inputs and outputs, respectively. Without loss of generality, the initial conditions not equal to zero can be viewed as $x_{0}(0)$ and $x(0)$.

This paper is aimed to propose fixed-time output feedback stabilizing controllers of the form

$\left\{\begin{array}{l}\dot{z}=\Theta_{o}(y, z), \quad z \in \mathbb{R}^{2}, \\ u_{0}=\Theta_{1}\left(x_{0}, z\right), \\ u_{1}=\Theta_{2}(y, z),\end{array}\right.$

such that system (3) under (4) is fixed-time stable. It should be noted that two output feedback controllers need to be constructed simultaneously, which brings challenges for choosing $u_{0}$ to fixed-time stabilize $x_{0^{-}}$ subsystem and $u_{1}$ to fixed-time stabilize $x$-subsystem, respectively.

Firstly, we formalize the form of the state feedback controller

$u_{0}=\operatorname{sign}\left(x_{0}(0)\|x\|\right) c+(1-\operatorname{sign}(\|x\|)) \varpi_{2}\left(x_{0}\right)$

with $c$ being a small positive constant and $\varpi_{2}\left(x_{0}\right)$ being continuous function determined later.

Remark 1 It should be noted that the initial condition $x(0)$ of $x$-subsystem is not equal to zero, which indicates switching controller $u_{0}=\operatorname{sign}\left(x_{0}(0)\|x\|\right) c$ being implemented until $\|x(t)\|=0, t>0$, that is, before achieving the fixed-time stabilization of $x$-subsystem, $u_{0}$ is a constant controller same as the sign of the initial value $x_{0}(0)$. Furthermore, after achieving the fixed-time stabilization of $x$-subsystem, switching controller $u_{0}=$ $\varpi_{2}\left(x_{0}\right)$ is used to fixed-time stabilize $x_{0}$-subsystem. 
Then, we reconsider $x$-subsystem

$\dot{x}_{1}=u_{0} x_{2}, \quad \dot{x}_{2}=u_{1}$

based on switching controller (5).

To achieve the fixed-time stabilization of system (6), state feedback controller $u_{1}$ is constructed as

$$
\begin{aligned}
u_{1}(x)= & -\left(k_{11}\left\lceil x_{1}\right\rfloor^{\gamma_{1}}+k_{12}\left\lceil x_{1}\right\rfloor+k_{13}\left\lceil x_{1}\right\rfloor^{\gamma_{1}^{\prime}}\right) \\
& -\left(k_{21}\left\lceil x_{2}\right\rfloor^{\gamma_{2}}+k_{22}\left\lceil x_{2}\right\rfloor+k_{23}\left\lceil x_{2}\right\rfloor^{\gamma_{2}^{\prime}}\right),
\end{aligned}
$$

where parameters $k_{i j}>0, i=1,2, j=1,2,3$ and $\gamma_{i}, \gamma_{i}, i=1,2$ are chosen as

$\gamma_{1}=\frac{\gamma}{2-\gamma}, \gamma_{2}=\gamma, \gamma_{1}^{\prime}=\frac{4-3 \gamma}{2-\gamma}, \gamma_{2}^{\prime}=\frac{4-3 \gamma}{3-2 \gamma}$

with $\gamma \in(0,1)$. Then, denoting $\Phi_{s}(x)=\left(u_{0} x_{2}, u_{1}\right)^{T}$ the vector field of system (6) under (7) and defining

$\Phi_{s 0}(x)=\left(u_{0} x_{2},-k_{11}\left\lceil x_{1}\right\rfloor^{\gamma_{1}}-k_{21}\left\lceil x_{2}\right\rfloor^{\gamma_{2}}\right)^{T}$,

$\Phi_{s \infty}(x)=\left(u_{0} x_{2},-k_{13}\left\lceil x_{1}\right\rfloor^{\gamma_{1}^{\prime}}-k_{23}\left\lceil x_{2}\right\rfloor^{\gamma_{2}^{\prime}}\right)^{T}$,

it is shown from (8) that $\forall \gamma \in(0,1), 0<\gamma_{i}<1<$ $\gamma_{i}^{\prime}, \quad i=1,2$ hold, which makes $\Phi_{s 0}(x)$ and $\Phi_{s \infty}(x)$ be regarded as approximating functions for $\Phi_{s}(x)$ in 0 -limit and $\infty$-limit, respectively. Furthermore, choosing $r_{s 0}=\left(\frac{2-\gamma}{1-\gamma}, \frac{1}{1-\gamma}\right)^{T}$, the vector field $\Phi_{s 0}(x)$ is $r_{s 0^{-}}$ homogeneous of degree $k_{s 0}=-1$. Similarly, the vector field $\Phi_{s \infty}(x)$ is $r_{s \infty}$-homogeneous of degree $k_{s \infty}=1$ with $r_{s \infty}=\left(\frac{2-\gamma}{1-\gamma}, \frac{3-2 \gamma}{1-\gamma}\right)^{T}$. Therefore, system 6 under (7) with parameters (8) is homogeneous in the $b i$-limit with $\left(r_{s 0}, k_{s 0}, \Phi_{s 0}(x)\right)$ and $\left(r_{s \infty}, k_{s \infty}, \Phi_{s \infty}(x)\right)$.

Proposition 1 Under controller (5), system (6) can be fixed-time stabilized by controller (7) with parameters given in (8), $\gamma \in(0,1)$ and $k_{i j}>0, i=1,2, j=1,2,3$.

Proof To prove the fixed-time stabilization of system (6), choose the Lyapunov function

$$
\begin{aligned}
W_{s}= & 2 k_{11}\left(\gamma_{1}^{\prime}+1\right)\left|x_{1}\right|^{\gamma_{1}+1}+k_{12}\left(\gamma_{1}+1\right)\left(\gamma_{1}^{\prime}+1\right)\left|x_{1}\right|^{2} \\
& +2 k_{13}\left(\gamma_{1}+1\right)\left|x_{1}\right|^{\gamma_{1}^{\prime}+1}+c\left(\gamma_{1}+1\right)\left(\gamma_{1}^{\prime}+1\right)\left|x_{2}\right|^{2}
\end{aligned}
$$

Obviously, $W_{s}$ is continuously differentiable, positive definite and radially unbounded. The derivative of $W_{s}$ can be derived by

$$
\begin{aligned}
\dot{W}_{s} \mid[6]= & k_{11} \Xi_{1}\left\lceil x_{1}\right\rfloor^{\gamma_{1}} \dot{x}_{1}+k_{12} \Xi_{1}\left\lceil x_{1}\right\rfloor \dot{x}_{1} \\
& +k_{13} \Xi_{1}\left\lceil x_{1}\right\rfloor^{\gamma_{1}^{\prime}} \dot{x}_{1}+c \Xi_{1}\left\lceil x_{2}\right\rfloor \dot{x}_{2}
\end{aligned}
$$

with $\Xi_{1}=2\left(\gamma_{1}+1\right)\left(\gamma_{1}^{\prime}+1\right)$. Substituting 6 and 7 into 12 yields

$$
\begin{aligned}
\left.\dot{W}_{s}\right|_{6}= & \Xi_{1} u_{0} x_{2}\left(k_{11}\left\lceil x_{1}\right\rfloor^{\gamma_{1}}+k_{12}\left\lceil x_{1}\right\rfloor+k_{13}\left\lceil x_{1}\right\rfloor^{\gamma_{1}^{\prime}}\right) \\
& -c \Xi_{1}\left\lceil x_{2}\right\rfloor\left(k_{11}\left\lceil x_{1}\right\rfloor^{\gamma_{1}}+k_{12}\left\lceil x_{1}\right\rfloor+k_{13}\left\lceil x_{1}\right\rfloor^{\gamma_{1}^{\prime}}\right. \\
& \left.+k_{21}\left\lceil x_{2}\right\rfloor^{\gamma_{2}}+k_{22}\left\lceil x_{2}\right\rfloor+k_{23}\left\lceil x_{2}\right\rfloor^{\gamma_{2}^{\prime}}\right) .
\end{aligned}
$$

Since $\left\lceil x_{2}\right\rfloor=x_{2}$ and $\left\lceil x_{2}\right\rfloor\left\lceil x_{2}\right\rfloor \varrho=\left|x_{2}\right|\left|x_{2}\right|^{\varrho}, \forall \varrho \geq 0$ hold, 13 can be rewritten as

$$
\begin{aligned}
\left.\dot{W}_{s}\right|_{6}= & \Xi_{1} u_{0}\left|x_{2}\right|\left(k_{11}\left|x_{1}\right|^{\gamma_{1}}+k_{12}\left|x_{1}\right|+k_{13}\left|x_{1}\right|^{\gamma_{1}^{\prime}}\right) \\
& -c \Xi_{1}\left|x_{2}\right|\left(k_{11}\left|x_{1}\right|^{\gamma_{1}}+k_{12}\left|x_{1}\right|+k_{13}\left|x_{1}\right|^{\gamma_{1}^{\prime}}\right) \\
& -c \Xi_{1}\left|x_{2}\right|\left(k_{21}\left|x_{2}\right|^{\gamma_{2}}+k_{22}\left|x_{2}\right|+k_{23}\left|x_{2}\right|^{\gamma_{2}^{\prime}}\right) .
\end{aligned}
$$

From Remark 1, we have $u_{0}=\operatorname{sign}\left(x_{0}(0)\|x\|\right) c$, which further indicates

$$
\left.\dot{W}_{s}\right|_{6} \leq-c \Xi_{1}\left|x_{2}\right|\left(k_{21}\left|x_{2}\right|^{\gamma_{2}}+k_{22}\left|x_{2}\right|+k_{23}\left|x_{2}\right|^{\gamma_{2}^{\prime}}\right) .
$$

It can be concluded that $\dot{W}_{s}$ in 14 is semi-negative definite. Moreover, $\left.\dot{W}_{s}\right|_{\sqrt{6}}=0$ implies that $x_{2}=0$. By LaSalle's invariance principle ( $[15]$ ), all the trajectories will converge to the invariant set $\Pi_{1}=\left\{\left(x_{1}, x_{2}\right) \mid x_{2}=\right.$ $0\}$. Together with system (6) with controller (7), we can conclude $x_{1}=0$ in $\Pi_{1}$. Therefore, the closed-loop system is asymptotically stable.

For $\dot{x}=\Phi_{s 0}(x)$ with $\Phi_{s 0}(x)$ given in (9), if the Lyapunov function is chosen as

$W_{s 0}=k_{11}\left|x_{1}\right|^{\gamma_{1}+1}+0.5 c\left(\gamma_{1}+1\right)\left|x_{2}\right|^{2}$,

and it can be easily calculated that

$\dot{W}_{s 0} \leq-c k_{21}\left(\gamma_{1}+1\right)\left|x_{2}\right|^{1+\gamma_{2}}$.

Similarly, if we choose

$W_{s \infty}=k_{13}\left|x_{1}\right|^{\gamma_{1}^{\prime}+1}+0.5 c\left(\gamma_{1}^{\prime}+1\right)\left|x_{2}\right|^{2}$

for $\dot{x}=\Phi_{s \infty}(x)$ with $\Phi_{s \infty}(x)$ given in 10$)$, it can be seen that

$\dot{W}_{s \infty} \leq-c k_{23}\left(\gamma_{1}^{\prime}+1\right)\left|x_{2}\right|^{\gamma_{2}^{\prime}+1}$

holds. By LaSalle's invariance principle, $\dot{x}=\Phi_{s 0}(x)$ and $\dot{x}=\Phi_{s \infty}(x)$ are asymptotically stable. Thus, from 1) of Lemma 1. we have proven Proposition 1. 


\subsection{Fixed-time state observer design}

In this section, for the case when only the system output signals $x_{0}$ and $x_{1}$ can be captured, the previous state controller (7) is not implementable since the unavailability of state information $x_{2}$. Thus, a new fixed-time observer for system 6 is proposed as follows

$\dot{z}_{1}=z_{2}+\bar{k}_{11}\left\lceil\zeta_{1}\right\rfloor^{\bar{\gamma}_{1}}+\bar{k}_{12}\left\lceil\zeta_{1}\right\rfloor+\bar{k}_{13}\left\lceil\zeta_{1}\right\rfloor^{\bar{\gamma}_{1}^{\prime}}$

$\dot{z}_{2}=u_{0} u_{1}+\bar{k}_{21}\left\lceil\zeta_{1}\right\rfloor^{\bar{\gamma}_{2}}+\bar{k}_{22}\left\lceil\zeta_{1}\right\rfloor+\bar{k}_{23}\left\lceil\zeta_{1}\right\rfloor^{\prime} \bar{\gamma}_{2}$

with $\zeta_{1}=x_{1}-z_{1}, \bar{k}_{i j}>0, i=1,2, j=1,2,3$ and parameters $\bar{\gamma}_{i}, \bar{\gamma}_{i}^{\prime}, i=1,2$ are given by

$\bar{\gamma}_{1}=\bar{\gamma}, \quad \bar{\gamma}_{2}=2 \bar{\gamma}-1, \quad \bar{\gamma}_{1}^{\prime}=2-\bar{\gamma}, \quad \bar{\gamma}_{2}^{\prime}=3-2 \bar{\gamma}$,

with $\bar{\gamma} \in\left(\frac{1}{2}, 1\right)$. From the structure of controller (5), it should be noted that controller (5) cannot be applied to the design of observer 15 directly. Thus, to solve this challenging problem, the controller $u_{0}$ is further reconstructed as

$u_{0}=\operatorname{sign}\left(x_{0}(0) \varpi_{1}(z)\right) c+\left(1-\operatorname{sign}\left(\varpi_{1}(z)\right)\right) \varpi_{2}\left(x_{0}\right)$,

where $\varpi_{1}(z)$ with $z=\left(z_{1}, z_{2}\right)^{T}$ and $\varpi_{2}\left(x_{0}\right)$ are continuous functions to be determined later.

To achieve to the fixed-time output feedback stabilization control objective, an appropriate function $\varpi_{1}(z)$ is chosen yielding the following proposition holding, whose rigorous proof can be seen in Appendix 6 .

Proposition 2 Under an appropriate $\varpi_{1}(z)$, switching controller (17) is a constant before achieving the fixed-time output feedback stabilization of system (6).

Then, based on Proposition 2, subtracting 15 from (6) yields

$$
\begin{aligned}
& \dot{\zeta}_{1}=-\bar{k}_{11}\left\lceil\zeta_{1}\right\rfloor^{\bar{\gamma}_{1}}-\bar{k}_{12}\left\lceil\zeta_{1}\right\rfloor-\bar{k}_{13}\left\lceil\zeta_{1}\right\rfloor^{\bar{\gamma}_{1}^{\prime}}+\zeta_{2}, \\
& \dot{\zeta}_{2}=-\bar{k}_{21}\left\lceil\zeta_{1}\right\rfloor^{\bar{\gamma}_{2}}-\bar{k}_{22}\left\lceil\zeta_{1}\right\rfloor-\bar{k}_{23}\left\lceil\zeta_{1}\right\rfloor^{\prime}{ }_{2}
\end{aligned}
$$

with $\zeta_{2}=u_{0} x_{2}-z_{2}$. The parameters in 16 are aimed to make system (18) be bi-limit homogeneous. To demonstrate this, let $\zeta=\left(\zeta_{1}, \zeta_{2}\right)^{T}$ and denote $\Phi_{p}(\zeta)$ be the vector field of system (18), which makes system (18) can be recorded as $\dot{\zeta}=\Phi_{p}(\zeta)$. Then, defining

$\Phi_{p 0}(\zeta)=\left(\bar{k}_{11}\left\lceil\zeta_{1}\right\rfloor^{\bar{\gamma}_{1}}+\zeta_{2},-\bar{k}_{21}\left\lceil\zeta_{1}\right\rfloor^{\bar{\gamma}_{2}}\right)^{T}$,

$\Phi_{p \infty}(\zeta)=\left(\bar{k}_{13}\left\lceil\zeta_{1}\right\rfloor^{\bar{\gamma}_{1}^{\prime}}+\zeta_{2},-\bar{k}_{23}\left\lceil\zeta_{1}\right\rfloor^{\bar{\gamma}_{2}^{\prime}}\right)^{T}$,

it can be seen that $\Phi_{p 0}(\zeta)$ is homogeneous with $k_{p 0}=$ -1 and $r_{p 0}=\left(\frac{1}{1-\bar{\gamma}}, \frac{\bar{\gamma}}{1-\bar{\gamma}}\right)^{T}$, and $\Phi_{p \infty}(\zeta)$ is homogeneous with $k_{p \infty}=1$ and $r_{p \infty}=\left(\frac{1}{1-\bar{\gamma}}, \frac{2-\bar{\gamma}}{1-\bar{\gamma}}\right)^{T}$. Since $\bar{\gamma} \in\left(\frac{1}{2}, 1\right), 0<\bar{\gamma}_{i}<1<\bar{\gamma}_{i}^{\prime}, i=1,2$ hold, the vector fields $\Phi_{p 0}(\zeta)$ and $\Phi_{p \infty}(\zeta)$ can be regarded as as approximating homogeneous functions for $\Phi_{p}(\zeta)$ in 0 -limit and $\infty$-limit, respectively. Therefore, the following Proposition can be summarized.

Proposition 3 Considering observer 15) with parameters given in (16), system (18) is fixed-time stabilized if $\bar{k}_{i j}>0, i=1,2, j=1,2,3$ with $\bar{\gamma} \in\left(\frac{1}{2}, 1\right)$.

Proof Choose a Lyapunov function

$$
\begin{aligned}
W_{o}= & \bar{k}_{21}(2-\bar{\gamma})\left|\zeta_{1}\right|^{2 \bar{\gamma}}+\bar{k}_{22} \bar{\gamma}(2-\bar{\gamma})\left|\zeta_{1}\right|^{2} \\
& +\bar{k}_{23} \bar{\gamma}\left|\zeta_{1}\right|^{4-2 \bar{\gamma}}+\bar{\gamma}(2-\bar{\gamma})\left|\zeta_{2}\right|^{2} .
\end{aligned}
$$

Obviously, $W_{o}$ in 21 is continuously differentiable, positive definite and radially unbounded for any $\bar{\gamma} \in$ $\left(\frac{1}{2}, 1\right)$. The derivative of $W_{o}$ can be derived by

$$
\left.\dot{W}_{o}\right|_{18}=\Xi_{2}\left(\bar{k}_{21}\left\lceil\zeta_{1}\right\rfloor^{2 \bar{\gamma}-1}+\bar{k}_{22}\left\lceil\zeta_{1}\right\rfloor+\bar{k}_{23}\left\lceil\zeta_{1}\right\rfloor^{3-2 \bar{\gamma}}\right) \dot{\zeta}_{1}
$$

with $\Xi_{2}=2 \bar{\gamma}(2-\bar{\gamma})$. Substituting $(18)$ into 22 results in

$$
\begin{aligned}
& \dot{W}_{o} \mid 18 \\
& =-\Xi_{2} \bar{k}_{21}\left(\bar{k}_{11}\left|\zeta_{1}\right|^{3 \bar{\gamma}-1}+\bar{k}_{12}\left|\zeta_{1}\right|^{2 \bar{\gamma}}+\bar{k}_{13}\left|\zeta_{1}\right|^{\bar{\gamma}+1}\right) \\
& -\Xi_{2} \bar{k}_{22}\left(\bar{k}_{11}\left|\zeta_{1}\right|^{1+\bar{\gamma}}+\bar{k}_{12}\left|\zeta_{1}\right|^{2}+\bar{k}_{13}\left|\zeta_{1}\right|^{3-\bar{\gamma}}\right) \\
& -\Xi_{2} \bar{k}_{23}\left(\bar{k}_{11}\left|\zeta_{1}\right|^{3-\bar{\gamma}}+\bar{k}_{12}\left|\zeta_{1}\right|^{4-2 \bar{\gamma}}+\bar{k}_{13}\left|\zeta_{1}\right|^{5-3 \bar{\gamma}}\right) \text {. }
\end{aligned}
$$

It can be concluded that $\dot{W}_{o}$ in 21 is semi-negative definite. Moreover, $\dot{W}_{o}|\sqrt{18}|=0$ implies that $\zeta_{1}=0$. By LaSalle's invariance principle, all the trajectories will converge to the invariant set $\Pi_{2}=\left\{\left(\zeta_{1}, \zeta_{2}\right) \mid \zeta_{1}=0\right\}$. Together with system (18), we can conclude $\zeta_{2}=0$ in $\Pi_{2}$. Therefore, system $(18)$ is asymptotically stable.

Choosing $\bar{k}_{i j}=0, i=1,2, j=2,3$ (or $\bar{k}_{i j}=$ $0, i, j=1,2)$ in 18 and $21-23$, system $\dot{\zeta}=\Phi_{p 0}(\zeta)$ (or $\dot{\zeta}=\Phi_{p \infty}(\zeta)$ ) can be asymptotically stabilized with $\Phi_{p 0}(\zeta)$ (or $\left.\Phi_{p \infty}(\zeta)\right)$ given in $(19)$ (or $(20)$ ).

Considering the $b i$-limit homogeneity of system (18) and by means of Lemma 1 system 18 is fixed-time stable.

\subsection{Fixed-time output feedback control}

Thus, it is time to construct fixed-time output feedback stabilizing controllers $u_{1}$ for system (6). Replacing $x_{2}$ in (7) as $z_{2} / u_{0}$ yields

$$
\begin{aligned}
u_{1}(z)= & -\left(k_{11}\left\lceil x_{1}\right\rfloor^{\gamma_{1}}+k_{12}\left\lceil x_{1}\right\rfloor+k_{13}\left\lceil x_{1}\right\rfloor^{\gamma_{1}^{\prime}}\right) \\
& -\left(k_{21}\left\lceil\frac{z_{2}}{u_{0}}\right\rfloor^{\gamma_{2}}+k_{22}\left\lceil\frac{z_{2}}{u_{0}}\right\rfloor+k_{23}\left\lceil\frac{z_{2}}{u_{0}}\right\rfloor^{\gamma_{2}^{\prime}}\right),
\end{aligned}
$$


where $x_{0}$ and $x_{1}$ are known outputs. Thus, the main results can be described in the following theorem.

Theorem 1 Under switching controller (17) determined by (28) and (32), and controller (24) with $z_{2}$ updated by (15), the closed-loop system (3), (15), (17) and (24) is fixed-time stable if $k_{i j}>0, \bar{k}_{i j}>0, i=1,2, j=1,2,3$ and $\gamma_{i}, \gamma_{i}^{\prime}, \bar{\gamma}_{i}, \bar{\gamma}_{i}^{\prime}, i=1,2$ are chosen as in Propositions 1 and 3 , respectively.

Proof Firstly, it follows from Proposition 2 that before achieving the fixed-time output feedback stabilization of system (6), the controller $u_{0}=\operatorname{sign}\left(x_{0}(0) \varpi_{1}(z)\right) c$ and then follows from Proposition 3 that there is a time $T_{1}>0$, independently of initial estimation error $\zeta_{1}(0)$ and $\zeta_{2}(0)$ such that $z_{2}(t)=u_{0}(t) x_{2}(t), \forall t \geq T_{1}$. Hence, controller 24) coincides with controller (7) for all $t \geq$ $T_{1}$.

Moreover, following Proposition 1, if all states of system (3) under (17) and (24) do not escape during $t \in$ $\left[0, T_{1}\right]$, there must be a time $T_{2}>0$, independently of $x_{1}\left(T_{1}\right), x_{2}\left(T_{1}\right)$ to achieve the fixed-time stabilization of system (6). Therefore, it is sufficient to prove Theorem 1 from the closed-loop system (3), 15) under (17) and (24) not escaping in finite time.

To achieve the proof, we reconsider the Lyapunov function $W_{s}$ in (11) whose derivative along the trajectory (6) under (17) and 24 is given by

$$
\left.\dot{W}_{s}\right|_{6} \leq-c \Xi_{1} x_{2}\left(k_{21}\left\lceil\frac{z_{2}}{u_{0}}\right\rfloor^{\gamma_{2}}+k_{22}\left\lceil\frac{z_{2}}{u_{0}}\right\rfloor+k_{23}\left\lceil\frac{z_{2}}{u_{0}}\right\rfloor^{\gamma_{2}^{\prime}}\right)
$$

with $\Xi_{1}=2\left(\gamma_{1}+1\right)\left(\gamma_{1}^{\prime}+1\right)$. By means of $z_{2}=u_{0} x_{2}-$ $\zeta_{2}, x_{2}\left\lceil\left.\frac{z_{2}}{u_{0}}\right|^{\varrho}=x_{2}\left|x_{2}-\frac{\zeta_{2}}{u_{0}}\right|^{\varrho} \operatorname{sign}\left(x_{2}-\frac{\zeta_{2}}{u_{0}}\right), \forall \varrho>0\right.$ can be easily obtained, yielding the following two different cases.

Case 1: If $\left|x_{2}\right|>\left|\frac{\zeta_{2}}{u_{0}}\right|, \operatorname{sign}\left(x_{2}-\frac{\zeta_{2}}{u_{0}}\right)=\operatorname{sign}\left(x_{2}\right)$ holds. Then, one has $x_{2}\left[\left.\frac{z_{2}}{u_{0}}\right|^{\varrho}=\left|x_{2}\right|\left|x_{2}-\frac{\zeta_{2}}{u_{0}}\right|^{\varrho}, \forall \varrho>\right.$ 0 and $\dot{W}_{s}$ in 25 can be rewritten as

$$
\begin{aligned}
& \dot{W}_{s} \mid\left[6 \leq-c \Xi_{1}\left|x_{2}\right|\left(k_{21}\left|x_{2}-\frac{\zeta_{2}}{u_{0}}\right|^{\gamma_{2}}+k_{22}\left|x_{2}-\frac{\zeta_{2}}{u_{0}}\right|\right.\right. \\
& \left.+k_{23}\left|x_{2}-\frac{\zeta_{2}}{u_{0}}\right|^{\gamma_{2}^{\prime}}\right) \text {. }
\end{aligned}
$$

Case 2: If $\left|x_{2}\right| \leq\left|\frac{\zeta_{2}}{u_{0}}\right|$, it is also easy to obtain $-x_{2}\left\lceil\left.\frac{z_{2}}{u_{0}}\right|^{\varrho}=\left|x_{2}\right|\left|x_{2}-\frac{\zeta_{2}}{u_{0}}\right|^{\varrho}, \forall \varrho>0\right.$, which indicates $\dot{W}_{s}$ in 25 satisfies

$$
\begin{aligned}
\left.\dot{W}_{s}\right|_{[6]} \leq & c \Xi_{1}\left|x_{2}\right|\left(k_{21}\left|x_{2}-\frac{\zeta_{2}}{u_{0}}\right|^{\gamma_{2}}+k_{22}\left|x_{2}-\frac{\zeta_{2}}{u_{0}}\right|\right. \\
& \left.+k_{23}\left|x_{2}-\frac{\zeta_{2}}{u_{0}}\right|^{\gamma_{2}^{\prime}}\right) .
\end{aligned}
$$

Then, we will confirm that for above two cases, there exists a constant $\Delta>0$ such that $\left.\dot{W}_{s}\right|_{6} \leq \Delta$ in time interval $\left[0, T_{1}\right]$. Firstly, from Proposition 3 , the fixedtime convergence of $\zeta_{2}$ can be gotten, which indicates $\zeta_{2}$ is bounded. Denoting $\Delta=\sup \left\{\Xi_{1}\left(\frac{k_{21} 2^{\gamma_{2}}}{c^{1+\gamma_{2}}}\left|\zeta_{2}\right|^{\gamma_{2}+1}+\right.\right.$ $\left.\left.\frac{2 k_{22}}{c}\left|\zeta_{2}\right|+\frac{k_{23} 2^{\prime} \gamma_{2}^{\prime}}{1+\gamma_{2}^{\prime}}\left|\zeta_{2}\right|^{\gamma_{2}^{\prime}+1}\right)\right\}, \Delta$ can be viewed as an upper bound of the right-hand side of (26) and (27), yielding $\left.\dot{W}_{s}\right|_{(6)} \leq \Delta$. Therefore, $W_{s}$ and system states $x_{1}$ and $x_{2}$ cannot escape in $\left[0, T_{1}\right]$. Moreover, from Proposition 3 observer states $z_{1}$ and $z_{2}$ also cannot escape in $\left[0, T_{1}\right]$.

Furthermore, since $u_{0}(t)=\operatorname{sign}\left(x_{0}(0) \varpi_{1}(z)\right) c, t \in$ $\left[0, T_{1}\right]$, we have $x_{0}(t) \leq x_{0}(0)+c t, t \in\left[0, T_{1}\right]$, which indicates the state $x_{0}$ cannot escape in $\left[0, T_{1}\right]$. Thus, the closed-loop system (6), (15) under (17) and (24) does not escape in $\left[0, T_{1}\right]$, which has proven that the closedloop system (6), (15) under (17) and $(24)$ is fixed-time stable.

Now, the fixed-time output feedback stabilization of $x_{0}$-subsystem is given below. Since the fixed-time output feedback stabilization of $x$-subsystem has been obtained, we have $x(t)=z(t)=u_{1}(t)=0, t \geq T_{2}$, which indicates the controller $u_{0}(t)=\varpi_{2}\left(x_{0}\right)$, for $t \geq$ $T_{2}+\tau$. Therefore, from 24 we construct

$\varpi_{2}\left(x_{0}\right)=-k_{11} x_{0}^{\gamma_{1}}-k_{13} x_{0}^{\gamma_{1}^{\prime}}$

to fixed-time stabilize $x_{0}$-subsystem with bounded initial condition $x_{0}\left(T_{2}+\tau\right)$.

Remark 2 If the initial condition $x_{0}(0)=0$, the switching controller (17) just needs to be rewritten into $u_{0}=$ $\operatorname{sign}\left(\varpi_{1}(z)\right) c+\left(1-\operatorname{sign}\left(\varpi_{1}(z)\right)\right) \varpi_{2}\left(x_{0}\right)$, and following Propositions 11 and 3 , and Theorem 1, the fixed-time output feedback stabilization of system (3) can also be obtained, which indicates the proposed control strategy is global sense.

Corollary 1 The output feedback control strategy proposed in Propositions 1 , 3 and Theorem 1 can fixed-time output feedback stabilize the following system

$\dot{x}_{0}=\left(1-\frac{\varepsilon^{2}}{2}\right) u_{0}$,

$\dot{x}_{1}=u_{0} x_{2}$,

$\dot{x}_{2}=u_{1}$,

where $\varepsilon$ is an unknown small bias in orientation, and satisfies $|\varepsilon| \leq \iota<1$ with $\iota$ being a known constant.

\section{An illustrative example}

Consider the bilinear model of a mobile robot with small-angle measurement error $12,14,23$, describing 
as

$\dot{x}_{l}=\left(1-\frac{\varepsilon^{2}}{2}\right) v$,

$\dot{y}_{l}=\theta_{l} v+\varepsilon v$,

$\dot{\theta}_{l}=\omega$,

with $\varepsilon \leq 1$ being a small bias in orientation, $\left(x_{l}, y_{l}, \theta_{l}\right)^{T}$ standing for the state of the locally approximate model, $v$ and $\omega$ being two inputs to denote the linear velocity and angular velocity, respectively. Taking the state transformation $x_{0}=x_{l}, x_{1}=y_{l}, x_{2}=\theta_{l}+\varepsilon, u_{0}=v$ and $u_{1}=\omega$, system 30 is transformed into system 29., where $\left(x_{0}, x_{1}, x_{2}\right)^{T}$ is state, $u_{0}$ and $u_{1}$ are inputs and $y=\left(x_{0}, x_{1}\right)^{T}$ is output, respectively.

Firstly, by virtue of Propositions 1 and 3 , and Theorem11. fixed-time output feedback stabilizing controllers are constructed as

$$
\left\{\begin{aligned}
\left\{\begin{array}{c}
\dot{z}_{1}=z_{2}+\left\lceil\zeta_{1}\right\rfloor \bar{\gamma}_{1}+\left\lceil\zeta_{1}\right\rfloor+\left\lceil\zeta_{1}\right\rfloor^{\prime} \\
\dot{z}_{2}=u_{0} u_{1}+\left\lceil\zeta_{1}\right\rfloor \bar{\gamma}_{2}+\left\lceil\zeta_{1}\right\rfloor+\left\lceil\zeta_{1}\right\rfloor \bar{\gamma}_{2}^{\prime}
\end{array}\right. \\
u_{0}=\operatorname{sign}\left(x_{0}(0) \varpi_{1}(z)\right) c+\left(1-\operatorname{sign}\left(\varpi_{1}(z)\right)\right) \varpi_{2}\left(x_{0}\right) \\
u_{1}=-\left(\left\lceil x_{1}\right\rfloor^{\gamma_{1}}+\left\lceil x_{1}\right\rfloor+\left\lceil x_{1}\right\rfloor^{\gamma_{1}^{\prime}}\right) \\
-\left(\left\lceil\frac{z_{2}}{u_{0}}\right\rfloor^{\gamma_{2}}+\left\lceil\frac{z_{2}}{u_{0}}\right\rfloor+\left\lceil\frac{z_{2}}{u_{0}}\right\rfloor^{\gamma_{2}^{\prime}}\right)
\end{aligned}\right.
$$

where $\zeta_{1}=x_{1}-z_{1}, \gamma=0.6$ and $\bar{\gamma}=0.6$, functions $\varpi_{1}(z)$ and $\varpi_{2}\left(x_{0}\right)$ are determined by 32 and 28 , respectively, and initial conditions $x_{0}(0)=15, x(0)=$ $(20,-10)^{T}$ and $z(s)=(5,5)^{T}, s \in[-\tau, 0]$ with $c=\tau=$ 0.5 . The numerical simulation result of the closed-loop system is shown in Fig 2

Remark 3 Due to $\operatorname{sign}(\cdot)$ being used in constructing $u_{0}$, a threshold of small value instead of absolute zero is applied, that is, let $\varpi_{1}(z) \approx 0$, when $\varpi_{1}(z) \leq 10^{-5}$ ( 32 ).

\section{Conclusion}

In this paper, a novel design approach for constructing fixed-time output feedback stabilizing controllers has been proposed for chained nonholonomic systems. Remarkably, the systems in question require two controllers to be constructed simultaneously, which are considerably different from those in the closely related literatures. In our design, by applying switching control technique, we first constructed a fixed-time output feedback stabilizing controller $u_{0}$ formally, and then based on this controller and by means of bi-limit homogeneous technique and the Lyapunov theorem, the fixedtime output feedback stabilizing controller $u_{1}$ based on fixed-time observer of $x$-subsystem are constructed. On the other hand, to obtain a fact, that is, before achieving the fixed-time output feedback stabilization of system (6), controller $u_{0}$ is a constant same as the sign of the initial condition of $x_{0}$-subsystem, a novel switching mechanism has been introduced, and then based on this mechanism, the fixed-time output feedback stabilizing controller $u_{0}$ is thus constructed precisely. Since the switching control strategy proposed for controller $u_{0}$ is hard to applied to chained nonholonomic systems with external disturbances, which is our one possible future study topic.

\section{Appendix}

In this section, we will give the detailed proof of Proposition 2, Firstly, from Remark 11 and Proposition 1, the fact that the achievement of fixed-time state feedback stabilization of system (6) implying $\|x(t)\|=0$, and vice versa. Moreover, since the state of $x_{2}$ cannot be measured, controller (5) should be reconstructed to be applied to fixed-time output feedback stabilize system (6), and an intuitive idea is to change $\|x\|$ in (5) as $\|z\|$.

However, the fact given in Section 3.1 will no longer hold, that is, the fixed-time output feedback stabilization of system (6) cannot be obtained from $\|z(t)\|=0$. In fact, it may occur the phenomenon that $\|z(t)\|=0$ happens infinite times during fixed-time observing the states of system (6), which will leads to the finite time $T_{1}$ not existing. Therefore, Proposition 3 will no longer hold. To avoid this phenomenon, we reconstruct the controller $u_{0}$ in (5) as (17) where $\varpi_{1}(z)$ is updated by

$\dot{\varpi}_{1}(z)=\|z(t)\|^{2}-\|z(t-\tau)\|^{2}$

with the initial condition $z(s) \neq 0, s \in[-\tau, 0]$.

Remark 4 Obviously, $\varpi_{1}(z)$ contains the information of $z(t)$ from time $t-\tau$ to $t$, which is critical to avoid the phenomenon that $\|z(t)\|=0$ happens infinite times during fixed-time observing the states of system (6).

Next, we will prove that under the choosing of $\varpi_{1}(z)$ in $(32$, the fact that before the achievement of fixedtime output feedback stabilization of system (6) implying $\varpi_{1}(z) \neq 0$.

For convenience, we divide the proof into three steps. In step 1 , we will provide that $\|z(t)\|, t \in(0,+\infty)$ is a continuous function. In step 2 , we will prove that the set $\Omega$ defined as $\Omega=\{t \mid\|z(t)\|=0, t>0\}$ is a discrete set. In step 3 , we prove that $\varpi_{1}(z)$ exists and $\varpi_{1}(z(t))>0$ before the achievement of fixed-time output feedback stabilization of system (6).

Step 1: Continuity analysis of $\|z(t)\|$ in $t \in(0,+\infty)$. 
Firstly, from the construction of observer 15 and controllers (17) and (24), we obtain that although $\dot{z}_{i}(t), t$ $(0, \infty), i=1,2$ are discontinuous at some points(where controllers (17) or (24) is discontinuous at these points), the functions $z_{i}(t), i=1,2$ are continuous in $(0, \infty)$. Then, based on the continuity of $z_{i}(t), t \in(0, \infty), i=$ 1,2 and by means of the definition of vector norm, we can directly prove the continuity of $\|z(t)\|$ in $t \in$ $(0,+\infty)$. Similarly, the continuity of $x_{i}(t), i=0,1,2$ and $\|x(t)\|$ in $t \in(0,+\infty)$ can be also obtained.

Step 2: Proof of discrete set $\Omega$.

Firstly, we give the definition of discrete set that is a set of points of a topological space such that each point in the set is an isolated point, i.e. a point that has a neighborhood that contains no other points of the set.

Next, the proof by contradiction method is applied to verify $\Omega$ is a discrete set. Firstly, we assume that there exists $t^{*} \in \Omega$ (the first one) is not an isolated point. There must exist a time sequence $\left\{t_{n}\right\}$ with $t_{n} \in$ $\Omega$ such that $t_{n} \rightarrow t^{*}$ as $n \rightarrow+\infty$ and without loss of generality, we assume that the time sequence $\left\{t_{n}\right\}$ tends to $t^{*}$ from the left hand.
Since $\left\|z\left(t^{*}\right)\right\|=0$, we have $z_{1}\left(t^{*}\right)=z_{2}\left(t^{*}\right)=0$. Fur$t \in$ thermore, $\left\|x\left(t^{*}\right)\right\| \neq 0$ can be also obtained, otherwise, the fixed-time output feedback stabilization of system (6) is already achieved, which contradicts the fact that before the achievement of fixed-time output feedback stabilization of system (6). Thus, based on $\left\|x\left(t^{*}\right)\right\| \neq 0$, we analyze the following three cases.

Case 1): $x_{1}\left(t^{*}\right) \neq 0$ and $x_{2}\left(t^{*}\right)=0$. When this case occurs, from observer 15 and controller (24), we obtain that at least $\dot{z}_{1}\left(t^{*}\right) \neq 0$. By means of the continuity of $x_{1}(t)$ in $t \in[0,+\infty)$, and based on local inheriting order property, there must exist a constant $\delta_{1}>0$, such that $\dot{z}_{1}(t) \neq 0, t \in \Omega_{1}=\left(t^{*}-\delta_{1}, t^{*}+\delta_{1}\right)$ and then we have $\left|z_{1}(t)\right|>0, t \in \Omega_{1} \backslash\left\{t^{*}\right\}$, which also indicates $\|z(t)\|>0, t \in \Omega_{1} \backslash\left\{t^{*}\right\}$.

On the other hand, since $t^{*} \in \Omega$ is not an isolated point and $t_{n} \rightarrow t^{*}$ as $n \rightarrow+\infty$, that is, for any constant $\delta_{2}>0$ choosing as $\delta_{2}<\delta_{1}$, there exists $N>0$, such that for all $n>N, t_{n} \in \Omega_{2}=\left(t^{*}-\delta_{2}, t^{*}\right)$, which contradicts $\|z(t)\|>0, t \in \Omega_{1} \backslash\left\{t^{*}\right\}$ (based on $\Omega_{2} \subseteq$ $\left.\Omega_{1}\right)$. Thus, $t^{*} \in \Omega$ is an isolated point, which has proven that $\Omega$ is a discrete set.
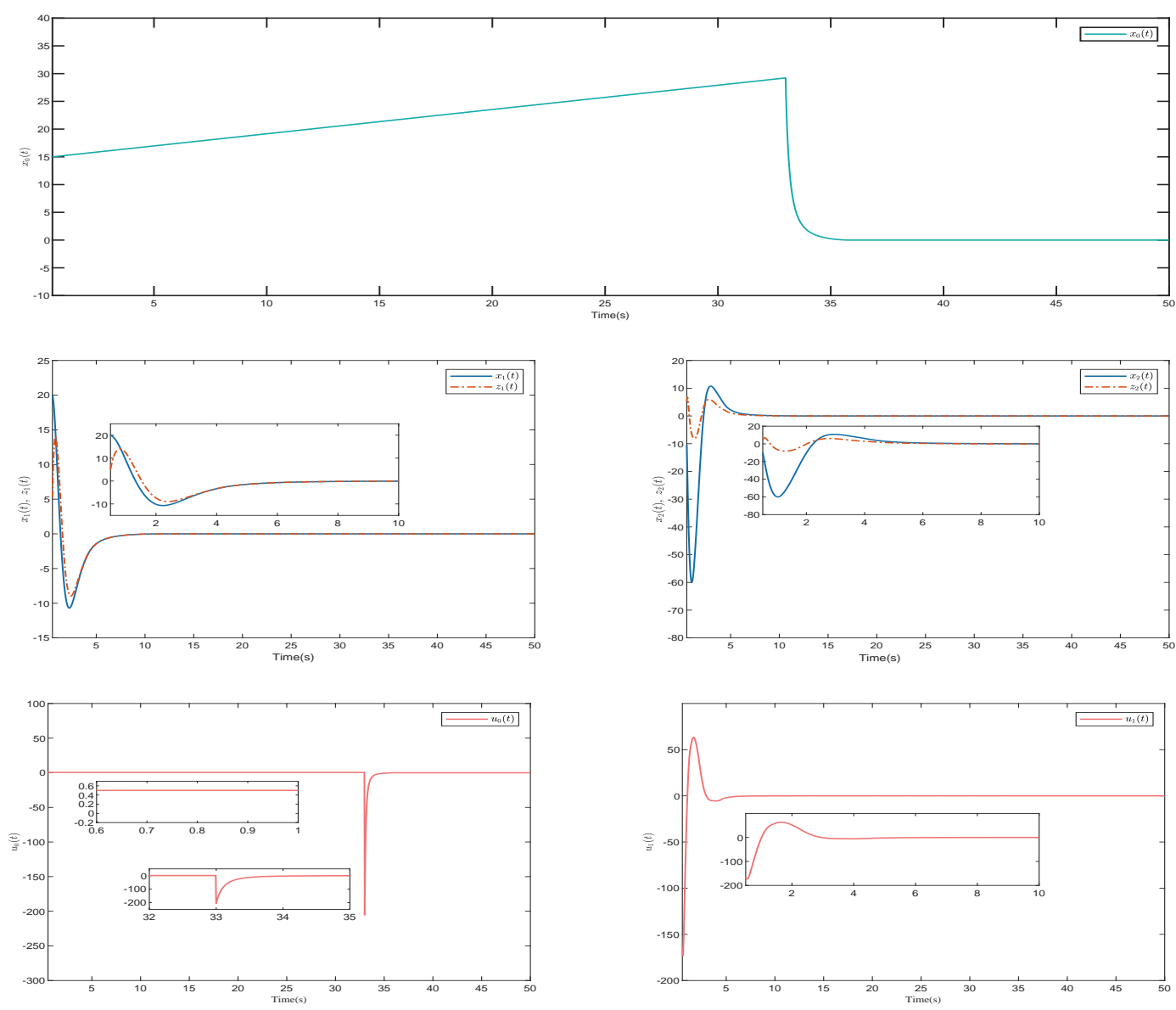

Fig. 2 Trajectories of the closed-loop system 28, 29, 31 and 32 with the small bias in orientation $\varepsilon=0.5$. 
Case 2): $x_{2}\left(t^{*}\right) \neq 0$ and $x_{1}\left(t^{*}\right)=0$. When this case occurs, we will firstly prove the state of $x_{0}\left(t^{*}\right) \neq 0$.

Since $t^{*} \in \Omega$ (the first one) is not an isolated point, thus, for any constant $\delta_{3}>0$, there must be finite points recorded as $t_{1}, \ldots, t_{M} \in \Omega_{3}=\left[0, t^{*}-\delta_{3}\right]$ such that $\left\|z\left(t_{k}\right)\right\|=0, k=1, \ldots, M$. Then, by means of the continuity of $\|z(t)\|, t \in \Omega_{3}$ and the initial condition $z(s) \neq 0, s \in[-\tau, 0]$, and from 32$]$, we have

$$
\begin{aligned}
\varpi_{1}(z(t))= & \int_{t-\tau}^{t}\|z(s)\|^{2} d s=\int_{t-\tau}^{t-t_{1}}\|z(s)\|^{2} d s+\cdots \\
& +\int_{t-t_{M}}^{t}\|z(s)\|^{2} d s>0
\end{aligned}
$$

which indicates the controller $u_{0}(t)=\operatorname{sign}\left(x_{0}(0) \varpi_{1}(z)\right) c$ and $\left|x_{0}(t)\right| \geq\left|x_{0}(0)\right|$ in $t \in \Omega_{3}$. Therefore, for any $\delta_{3}$, if $x_{0}\left(t^{*}\right)=0$, there exists $\varepsilon_{3}=\frac{\left|x_{0}(0)\right|}{2}$, such that when $\left|t^{*}-\left(t^{*}-\delta_{3}\right)\right| \leq \delta_{3},\left|x_{0}\left(t^{*}\right)-x_{0}\left(t^{*}-\delta_{3}\right)\right| \geq \varepsilon_{3}=$ $\frac{\left|x_{0}(0)\right|}{2}$ holds, which contradicts the continuity of $x_{0}(t)$ in $t \in[0,+\infty)$. Thus, we have proven $x_{0}\left(t^{*}\right) \neq 0$, and similarly, $x_{0}(t) \neq 0, t \in \Omega_{4}=\left(t^{*}-\delta_{3}, t^{*}+\delta_{3}\right)$ can also be obtained, which also indicates the controller $u_{0}(t) \neq 0, t \in \Omega_{4}$.

Next, from system (6) with $u_{0}(t) \neq 0, t \in \Omega_{4}$ and $x_{2}\left(t^{*}\right) \neq 0$, we have $\dot{x}_{1}(t) \neq 0, t \in \Omega_{4}$ and based on local inheriting order property, there is a constant $\delta_{4}>$ 0 such that $x_{1}(t) \neq 0, t \in \Omega_{5}=\left(t^{*}-\delta_{4}, t^{*}+\delta_{4}\right) \backslash\left\{t^{*}\right\}$. Then, from Case 1), we have proven that $t^{*} \in \Omega$ is an isolated point and $\Omega$ is a discrete set.

Case 3): $x_{1}\left(t^{*}\right) \neq 0$ and $x_{2}\left(t^{*}\right) \neq 0$. When this case occurs, combining with Case 1) and Case 2), we have proven that $t^{*} \in \Omega$ is an isolated point and $\Omega$ is a discrete set. This completes the proof.

Step 3. Proof of $\varpi_{1}(z(t))>0$.

From Step 1, we have $\|z(t)\|$ is continuous in $t \in$ $(0,+\infty)$, and since $\Omega$ is a discrete set, and then, for any given $t>0$, there exist finite points recorded as $t_{1}, \ldots, t_{N} \in[t-\tau, t]$ such that $\left\|z\left(t_{k}\right)\right\|=0, k=1, \ldots, N$. Therefore, $\varpi_{1}(z(t))$ defined in (32) exists and satisfies

$$
\begin{aligned}
\varpi_{1}(z(t))= & \int_{t-\tau}^{t}\|z(s)\|^{2} d s=\int_{t-\tau}^{t-t_{1}}\|z(s)\|^{2} d s+\cdots \\
& +\int_{t-t_{N}}^{t}\|z(s)\|^{2} d s>0 .
\end{aligned}
$$

Thus, by means of the structure of controller (17), we have proven (17) is a constant controller before the achievement of fixed-time output feedback stabilization of system (6). This completes the proof.

Acknowledgements The work was supported by the National Natural Science Foundation of China (61973189), the Research Fund for the Taishan Scholar Project of Shandong Province of China (ts20190905), and the Natural Science Foundation of Shandong Province of China (ZR2020ZD25).

\section{Compliance with ethical standards}

Conflict of interest The authors declare that they have no conflict of interest.

\section{References}

1. Andrieu, V., Praly, L.: On the existence of a kazantzis-kravaris/luenberger observer. SIAM Journal on Control and Optimization 45(2), 432-456 (2006)

2. Andrieu, V., Praly, L., Astolfi, A.: Homogeneous approximation, recursive observer design, and output feedback. SIAM Journal on Control and Optimization 47(4), 18141850 (2008)

3. Bacciotti, A., Rosier, L.: Lyapunov Functions and Stability in Control Theory. Springer (2005)

4. Chen, C.: A unified approach to finite-time stabilization of high-order nonlinear systems with and without an output constraint. International Journal of Robust and Nonlinear Control 29, 393-407 (2019)

5. Defoort, M., Demesure, G., Zuo, Z., Polyakov, A., Djemai, M.: Fixed-time stabilisation and consensus of nonholonomic systems. IET Control Theory \& Applications 10(18), 2497-2505 (2016)

6. Ding, S., Park, J., Chen, C.: Second-order sliding mode controller design with output constraint. Automatica 112(108704) (2020)

7. Du, H., Wen, G., Wu, D., Cheng, Y., Lü, J.: Distributed fixed-time consensus for nonlinear heterogeneous multiagent systems. Automatica 113, 1-11 (2020). DOI https: //doi.org/10.1016/j.automatica.2019.108797

8. Esfandiari, F., Khalil, H.: Output feedback stabilization of fully linearizable systems. International Journal of Control 56(5), 1007-1037 (1992)

9. Filippov, A.: Differential Equations with Discontinuous Right Hand Sides. Dordrecht, The Netherlands: Kluwer Academic Publishers (1988)

10. Gao, F., Wu, Y., Li, H., Liu, Y.: Finite-time stabilisation for a class of output-constrained nonholonomic systems with its application. International Journal of Systems Science 49(10), 2155-2169 (2018)

11. Gao, F., Wu, Y., Zhang, Z., Liu, Y.: Global fixed-time stabilization for a class of switched nonlinear systems with general powers and its application. Nonlinear Analysis: Hybrid Systems 31, 56-68 (2019)

12. Ge, S., Wang, Z., Lee, T.: Adaptive stabilization of uncertain nonholonomic systems by state and output feedback. Automatica 39(8), 1451-1460 (2003)

13. Hua, C., Li, Y., Guan, X.: Finite/fixed-time stabilization for nonlinear interconnected systems with dead-zone input. IEEE Transactions on Automatic Control 62(5), 2554-2560 (2017)

14. Jiang, Z.: Robust exponential regulation of nonholonomic systems with uncertainties. Automatica 36, 189-209 (2000)

15. Khalil, H.: Nonlinear Systems (3rd ed.). Upper Saddle River, NJ: Prentice Hall. (2005)

16. Levant, A.: Homogeneity approach to high-order sliding mode design. Automatica 41(5), 823-830 (2005)

17. Lin, W., Pongvuthithum, R., Qian, C.: Control of highorder nonholonomic systems in power chained form using discontinuous feedback. IEEE Transactions on Automatic Control 47(1), 108-115 (2002) 
18. Liu, Y., Jing, Y., Liu, X., Li, X.: Survey on finite-time control for nonlinear systems. Control Theory and Applications 37(1), 1-12 (2020)

19. Lopez-Ramirez, F., Efimov, D., Polyakov, A., Perruquetti, W.: Fixed-time output stabilization and fixedtime estimation of a chain of integrators. International Journal of Robust and Nonlinear Control 28, 4647-4665 (2018)

20. Lopez-Ramirez, F., Polyakov, A., Efimov, D., Perruquetti, W.: Finite-time and fixed-time observer design: Implicit lyapunov function approach. Automatica 87, 52-60 (2018)

21. Mazenca, F., Bernard, O.: Interval observers for linear time-invariant systems with disturbances. Automatica 47(1), 140-147 (2011)

22. Ménard, T., Moulay, E., Perruquetti, W.: Fixed-time observer with simple gains for uncertain systems. Automatica 81, 438-446 (2017)

23. Ning, B., Han, Q.: Prescribed finite-time consensus tracking for multi-agent systems with nonholonomic chainedform dynamics. IEEE Transactions on Automatic Control 64(4), 1686-1693 (2019)

24. Polyakov, A.: Nonlinear feedback design for fixed-time stabilization of linear control systems. IEEE Transactions on Automatic Control 57(8), 2106-2110 (2012)

25. Polyakov, A.: Generalized Homogeneity in Systems and Control. Springer (2020)

26. Polyakov, A., Efimov, D., Perruquetti, W.: Finite-time and fixed-time stabilization: Implicit lyapunov function approach. Automatica 51, 332-340 (2015)

27. Ríos, H., Teel, A.: A hybrid observer for fixed-time state estimation of linear systems. In: In 2016 IEEE 55th Conference on Decision and Control, pp. 5408-5413 (2016)

28. Shen, Y., Xia, X.: Semi-global finite-time observers for nonlinear systems. Automatica 44(12), 3152-3156 (2008)

29. Tian, B., Zuo, Z., Yan, X., Wang, H.: A fixed-time output feedback control scheme for double integrator systems. Automatica 80, 17-24 (2017)

30. Yao, H., Gao, F., Huang, J., Wu, Y.: Barrier lyapunov functions-based fixed-time stabilization of nonholonomic systems with unmatched uncertainties and time-varying output constraints. Nonlinear Dynamics (2020). DOI https://doi.org/10.1007/s11071-019-05450-3

31. Yuan, J., Ding, S., Mei, K.: Fixed-time sosm controller design with output constraint. Nonlinear Dynamics 102, 1567-1583 (2020)

32. Zhang, X., Feng, G., Sun, Y.: Finite-time stabilization by state feedback control for a class of time-varying nonlinear systems. Automatica 48(3), 499-504 (2012)

33. Zhang, Z., Liu, X., Liu, Y., Lin, C., Chen, B.: Fixed-time almost disturbance decoupling of nonlinear time-varying systems with multiple disturbances and dead-zone input. Information Sciences 450, 267-283 (2018)

34. Zuo, Z., Han, Q., Ning, B., Ge, X., Zhang, X.: An overview of recent advances in fixed-time cooperative control of multiagent systems. IEEE Transactions on Industrial Informatics 14(6), 2322-2334 (2018) 
Figures

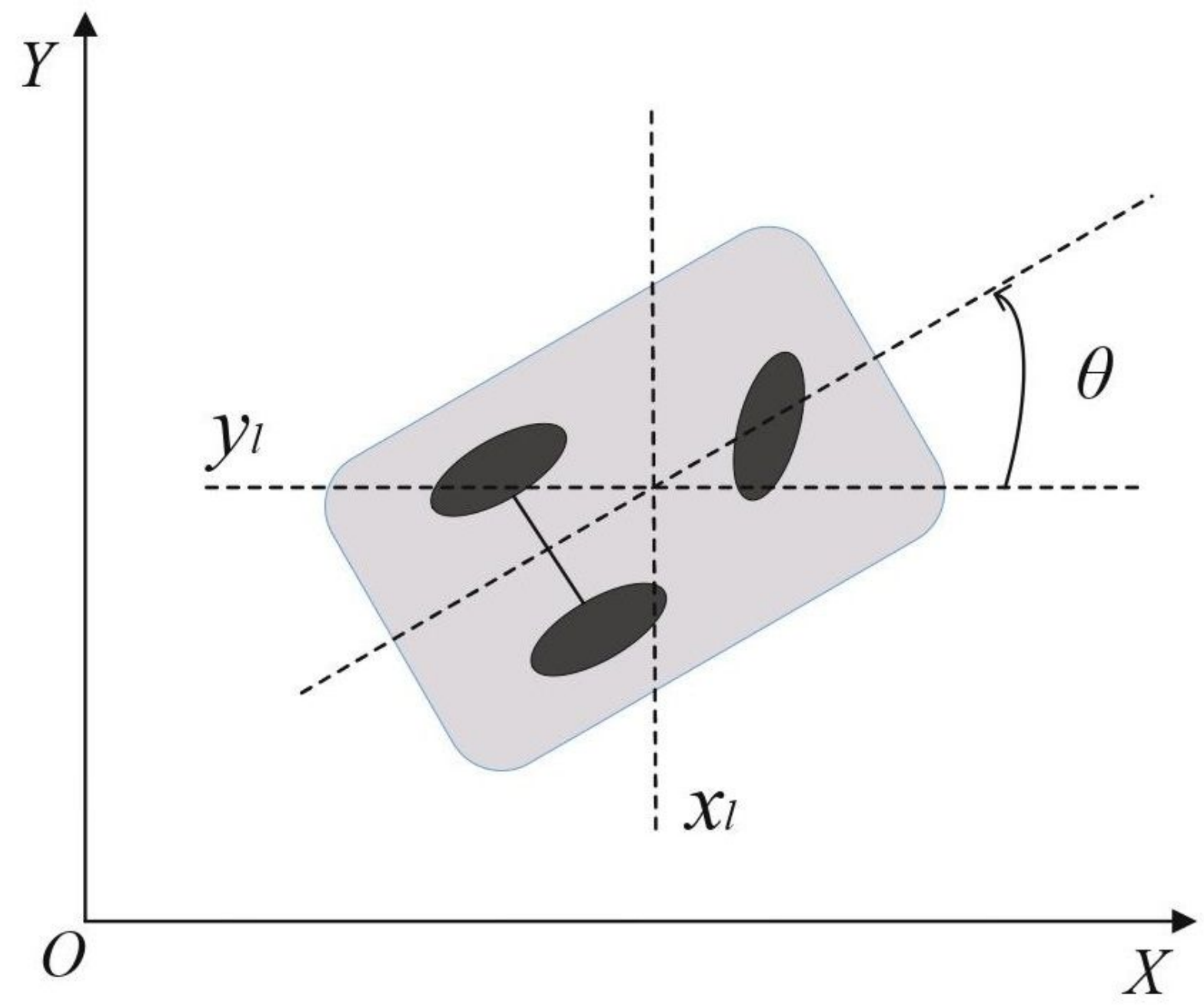

Figure 1

The planar graph of a unicycle-type mobile robot. 

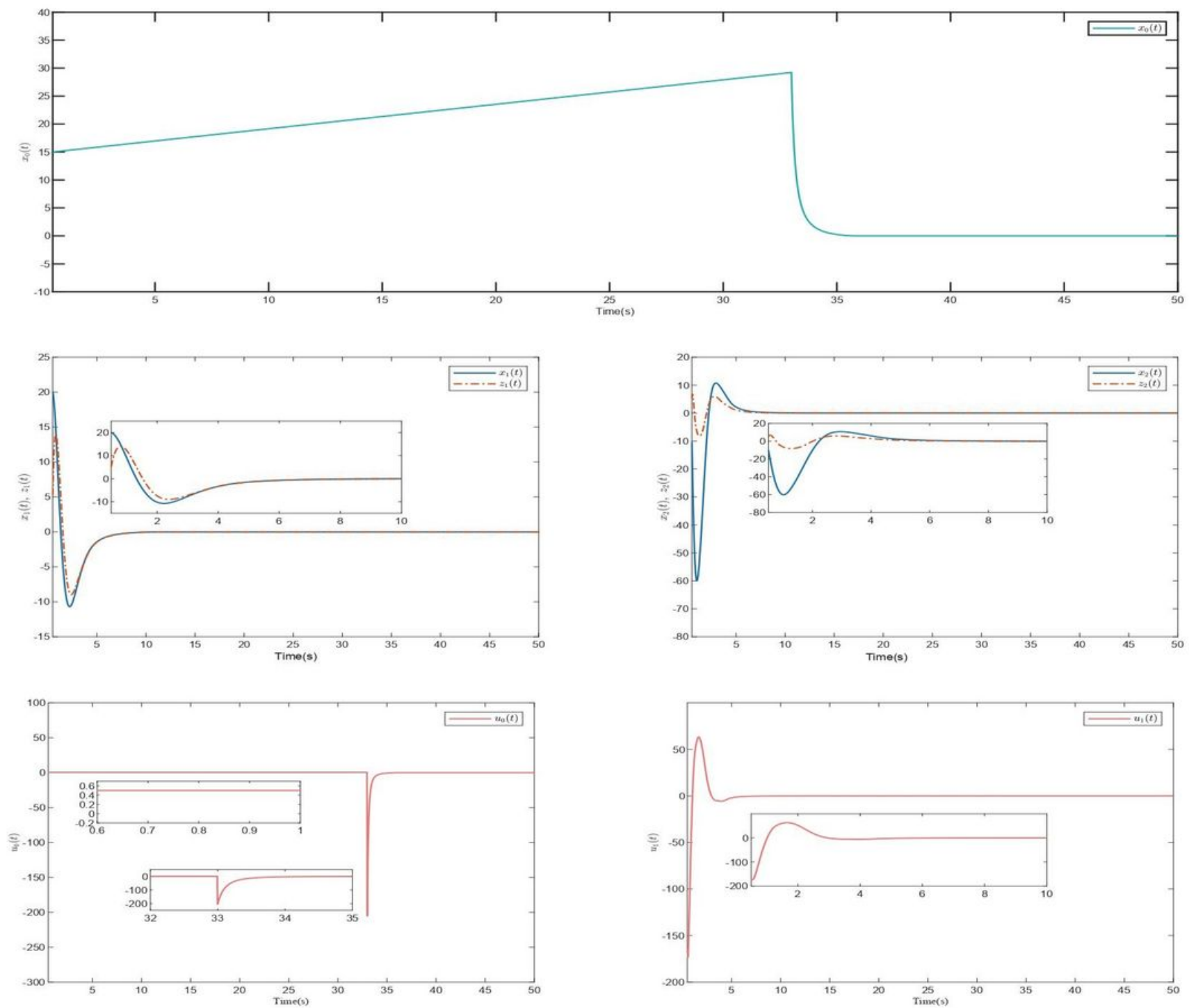

Figure 2

Trajectories of the closed-loop system (28), (29), (31) and (32) with the small bias in orientation $\varepsilon=0.5$. 